\title{
Lieux de vente de vin de palme et débats publics : une autre face de l'espace public à Kisangani
}

\author{
John Nkoko Lipambala*
}

\begin{abstract}
Résumé
A Kisangani, les conditions socio-économiques défavorables poussent certains individus à se défouler dans les lieux de consommation de vin de palme appelé « libondo » en jargon local. Ces lieux sont devenus des sites où les hommes et les femmes se rencontrent après le marché, le service ou les travaux de champs. Au-delà de la prise du vin de palme dont le coût répond bien à la bourse de chacune d'entre elles, les personnes qui fréquentent ces lieux profitent de l'ambiance qui y règne pour s'exprimer sur les grandes questions de la ville, de la province, du pays voire même de la planète. Car, les bonnes informations, estiment-elles, sont celles qui sont transmises dans de tels endroits.

Ainsi, les points de vente de « libondo » sont devenus à Kisangani des espaces publics où s'organisent les débats et discussions, de façon spontanée et sans aucune formalité, sur les questions d'actualité. Tout peut donc se dire dans ces sites et il n'existe pas de sujet tabou ni de contrainte sécuritaire. Cela parce qu'on se croit être en face de celui ou de celle avec qui on partage le même niveau de vie et par conséquent les mêmes difficultés. L’on ne devrait donc pas se créer de problèmes ; au contraire on doit considérer ce cadre comme la seule chance où la libre expression est permise.
\end{abstract}

\section{Abstract}

In Kisangani, unfavourable socioeconomic conditions lead some people to let off steam in premises where palm wine, known as libondo in the local jargon, is consumed. Such premises have become places where men and women meet after market, after work or after they have

\footnotetext{
* Enseignant et chercheur à l’Université de Kisangani, DRC.
}

Email :ijdlipamala@hotmail.com 
finished working in the fields. In addition to drinking palm wine, whose cost fits every budget, the people who frequent these places take advantage of the atmosphere that prevails there to express their views on the major issues of the city, the province, the country or even the planet. In their view, the right information is passed on in such places.

Thus, in Kisangani, points of sale of libondo have become public spaces where debates and discussions are organized, spontaneously and informally, on topical issues. Anything can be said in these places and there are no taboo subjects or security constraints. This is because the people there believe they are with others who share the same standard of living and thus the same hardships. Accordingly, they should not cause problems for one another; on the contrary, these places must be seen as the only chance for freedom of expression.

\section{Introduction}

L'activité politique est devenue un métier qui n'inspire plus confiance pour une certaine catégorie d'individus en République Démocratique du Congo. En effet, les discours ne sont pas traduits en actes, ce qui crée souvent un sentiment de méfiance des gouvernés envers les gouvernants.

Cette méfiance est d'autant plus accentuée par le fait que les sources d'informations officielles (médias) sont instrumentalisées par le pouvoir. Aussi la censure est-elle devenue un frein à la liberté d'expression, l'un des piliers de la démocratie. Le verrouillage de l'espace politique a donc entraîné un fort contrôle de l'expression politique. En dépit du fait que la République Démocratique du Congo compte environ une centaine de chaînes de radio et télévision, la liberté d'expression reste encore un effort à faire dans le chef de la classe politique, ce qui s'explique pendant le régime de la deuxième République sous Mobutu par les arrestations des journalistes et la valorisation du culte de la personne.Cette situation semble ne pas disparaître actuellement, comme en témoigne le rapport annuel 2010 de l'organisation Journalistes en Danger(JED), une Organisation qui lutte pour la liberté de la presse et la protection des journalistes en République démocratique du Congo.

Les crises politiques qu’a connues la République Démocratique du Congo ont eu des conséquences économiques sans précédent, poussant ainsi la population à développer certains -mécanismes de survie et d'adaptation. Le commerce informel peut être cité parmi ces activités de survie et d'adaptation. Ces adaptations se sont élargies sur tous les secteurs de la vie de la population congolaise.

A Kisangani, la population n'est pas restée sans innover ou s'adapter aux conditions socioéconomiques difficiles qu'a traversées et continue de traverser toute la République. En effet, le prix des boissons industrielles ne permet plus à tous les consommateurs de s'en procurer. Le vin de palme 
joue à cette fin un rôle de substitution parmi tant d'autres boissons artisanales. Les lieux de vente de vin de palme ${ }^{1}$ sont devenus des endroits où les gens se rencontrent après le travail, le marché ou toute autre activité de la journée.

Ces sites de rassemblement sont transformés en véritables endroits d'échanges, de discussions et de débats sur les grandes questions de société touchant à la politique, à l'économie, à la culture, etc.

Au-delà de la fonction manifeste qui est la consommation de vin de palme, se développe une fonction latente qui devient la plus importante ${ }^{2}$. En effet, ces endroits attirent une grande foule non seulement pour la consommation de la boisson, mais surtout pour des discussions et échanges qui s’y déroulent. Ces sites permettent donc le passage d'un espace commun à un espace public qui implique les débats et discussions autour de certains thèmes. Le pouvoir ne réglemente pas le fonctionnement ni la structure de ces sites. Il les tolère dans la mesure où les activités qui s'y déroulent ne constituent pas un danger pour la sécurité publique.

Pour les usagers de ces sites, l'ambiance qui y règne constitue un moment propice pour s'exprimer sur n'importe quelle question de la ville, de la province, du pays, voire de la planète.

Les points de vente de "libondo » sont donc des espaces publics où s'organisent les débats et discussions de façon spontanée et sans formalités, ni contrôle du pouvoir en place. Tout sujet peut y être discuté au tour du verre, sans inquiétude ni peur. Ces cadres sont considérés par une certaine tranche de la population comme les seuls vrais espaces publics où la liberté d'expression est garantie.

La présente contribution se veut une analyse fonctionnelle de ces sites. Il s'agit de porter un regard sur une politologie de la quotidienneté pour comprendre ces sites comme une innovation/adaptation de l'espace public dans le contexte local de la ville de Kisangani, à côté bien sûr d'autres espaces publics qui sont les médias, les espaces verts et autres. Il s'agit ici d'une approche par le banal, l'anodin. Sans céder à la tentation de sociologiser des épiphénomènes, l'ambition est de décrypter les événements à la lumière de tout ce qui ne se dit pas, et des choses que l'on n'énonce pas forcément de manière explicite (Monga 1994:10).

Nous répondrons tour à tour aux questions de savoir les usagers et les raisons d'émergence de ces sites, la prise en charge de la salubrité de ces sites, des thèmes débattus dans les lieux de consommation de vin de palme, des sources et problème de la dénaturalisation des informations. Nous ne manquerons pas d'aborder la question de l'expression de l'opinion publique dans ces sites. 


\section{Raisons d'émergences et qualités des usagers des points de vente de vin de palme à Kisangani}

Avant d'aborder la question relative aux raisons qui ont joué en faveur de l'essor de ces sites, nous voulons bien retracer de manière plus ou moins succincte l'organisation de ces sites. En effet, ces sites sont pour la majorité des cas exposés en plein air, soit à l'ombre des arbres ou encore sous les paillotes. Les activités de ces sites ne commencent pas aux mêmes heures, les unes commencent plutôt que les autres et ferment également un peu plus tôt. De façon générale, les activités commencent dans l'après-midi ; ce qui montre que l'afflux vers ces endroits paraît répondre à l'organisation sociale. Cette dernière exige que la matinée soit consacrée au travail de toute sorte ${ }^{3}$ pour que le soir, chacun arrive à se distraire au tour d'un verre. La quête de survie constitue la préoccupation journalière de chaque congolais. Dans ces sites, les buveurs ou usagers sont rangés souvent en forme de rectangle, de cercle ou de carré pour une bonne disposition aux discussions et débats. Quelques chaises et bancs servent de sièges pour certains sites. Dans d'autres sites, c'est plutôt les bambous installés en forme de banc qui constituent les sièges, rappelant ainsi l'usage de l'arbre à palabres dans les villages. Ces arbres à palabres pouvaient être compris également dans la logique des espaces où les grandes questions du village étaient débattues ; on était là dans un espace public.

Si ces lieux sont des endroits de divertissement de manière manifeste, nous disons que la façon dont les sièges y sont arrangés prédispose à des échanges et débats, bien que cela ne soit pas la raison première de leur existence.

La combinaison des éléments de la modernité (on est en pleine ville avec toutes ses exigences) à ceux de la tradition (le vin de palme étant une boisson traditionnelle dont la consommation devrait en principe diminuer ou tendre vers la disparition en ville) nous pousse à soutenir l'idée selon laquelle ces sites essaient de faire une symbiose des espaces publics modernes à ceux traditionnels, pour autant que la tradition ne disparaisse pas totalement en ville. Elle subit les influences de la modernité sans disparaître pour autant.

De par son utilisation, Habermas comprenait par espace public le rassemblement des personnes privées faisant usage public de leur raison (Alcaud et Bouvet 2004:111). Il faisait allusion à la classe bourgeoise.

L'espace public a pu s'incarner dans une série de lieux réels (cafés littéraires anglais, salons et clubs français, sociétés d'érudits allemands) où une fraction restreinte de la population, les bourgeois cultivés, se rencontrait pour discuter, avec une certaine liberté de ton et, aussi, une volonté de savoir et de comprendre, caractéristique des Lumières, les 
œuvres de l'esprit de l'époque, mais aussi peu à peu les décisions du pouvoir (Alcaud et Bouvet 2004:111)

L’on s’aperçoit bien qu'il s’agissait de rassemblements de personnes privées, mais dans lesquels les affaires publiques étaient traitées, ne transformant pas pour autant ces personnes privées en des personnalités publiques. La notion a évolué par la suite pour devenir de nos jours un des justificatifs de la démocratie. On ne peut parler actuellement de la démocratie sans qu’on fasse allusion à l'espace public. En effet, Alain Caillé précise, en ce qui concerne l’idéal démocratique, que ce qui importe, c'est l'existence d'espaces publics dans lesquels les sujets puissent débattre, former une opinion et conquérir la reconnaissance de leur valeur et la certitude d'exister légitimement et valablement aux yeux des autres. La société démocratique est d’abord une société plurielle et pluraliste (Caillé 2004:32-33).

Il ne peut y avoir démocratie sans débats ni échanges d’idées dans les endroits spécifiques.

L’idée défendue selon laquelle la communication doit impliquer tous les sujets rationnels se matérialise dans les conversations au sein de certains cadres bien appropriés, les espaces publics. L’idéal de communication impliquant tous les sujets rationnels et entièrement dénués de domination et d’intérêts demeure un enjeu important des ces espaces publics.

A Kisangani, les raisons économiques sont également à la base de l'émergence de ces sites. En effet, le vin de palme reste la boisson dont le prix n'est pas à craindre. Il est à la portée de la bourse d'une bonne partie de la population boyomaise ${ }^{4}$. Tout le monde peut en consommer quant à son coût et à la disponibilité sur le marché. Aussi la crise de l'Etat congolais, couplée de guerres, a-t-elle été un élément qui a appuyé sur l'accélérateur de l'essor de ces endroits. Les bas salaires, l'absence d'encadrement et le mauvais fonctionnement de l'Etat ont jeté dans les rues des villes ceux qui ne pouvaient joindre les deux bouts avec les rémunérations officielles (Kanyarwunga 2008:341).

La modicité des salaires des fonctionnaires, le non-paiement de la bourse des étudiants et le taux élevé de chômeurs sont les facteurs favorables à l'émergence de ces sites comme non seulement lieux de jouissance, mais surtout des endroits de défoulement pour une vie urbaine quasi intenable.

Lorsque le fonctionnaire n’arrive pas à s’acheter régulièrement une bière qui lui demande de longues réflexions, il se contente d'aller chercher une bouteille de vin de palme qui ne dépasse généralement pas la moitié du coût de la bière. Sa soif étanchée lui fait croire qu'il a pu vivre comme toute personne capable de satisfaire ses besoins. Bien qu'artisanal, le vin de palme a des effets aphrodisiaques comparables à la bière produite industriellement. A ce sujet Steward précise : 
La production de vin de palme est dite artisanale à cause de la petite échelle des exploitants, aux techniques ou technologies appropriées et aux matériels utilisés qui, du reste, sont presque rudimentaires, et aux capitaux faibles dont disposent les exploitants ou les producteurs qui n’ont jamais été des entrepreneurs auparavant, contrairement au secteur industriel moderne ou semi moderne tendant à faire appel à des échelles relativement vastes et immobilisant d'importants capitaux (Steward 1974:32).

Le caractère artisanal de ce vin a certes des répercussions sur son prix qui, du reste, appelle un grand nombre de consommateurs dans un environnement sociopolitique et économique de crise et d'incertitude. Faute de mieux, le vin de palme devient une boisson de substitution pour ceux qui s'étaient habitués à fréquenter les bars et autres endroits modernes de prise de boisson, dont les boîtes de nuit et les snack-bars.

Ainsi, les sites de vin de palme attirent de façon voulue ou non les personnes de toutes les catégories sociales dont le revenu faible constitue le dénominateur commun. Les universitaires sont à compter dans le rang des usagers dans la mesure où ils ne sont pas traités par l'Etat à leur juste valeur. Les étudiants se comptent en grand nombre à cause des conditions dans lesquelles ils sont exposés par le pouvoir depuis longtemps. Les fonctionnaires se retrouvent dans ces endroits pour étancher leur soif de la bière dont ils ne sont plus capables de supporter le coût. Il faut également ajouter les sansemploi et autres catégories qui, au long de la journée, s'adonnent à la débrouillardise. Ils se retrouvent le soir dans ces sites pour ainsi chercher une consolation au tour de l'ivresse. Car une certaine opinion soutient que pendant l'état d'ivresse, on oublie ce qu'on est. Cela est acceptable pour certaines personnes. Il sied de préciser que cet état n'est que passager et peut également conduire à des actes susceptibles de violer les règles de bonne conduite et de la moralité, ce qui arrive quelquefois dans ces sites.

Le caractère hétérogène des usagers de ces points de vente joue également à l'enrichissement des débats et discussions. Les uns et les autres abordent différents thèmes et les interventions sont spontanées. Cependant, il se crée une certaine autorité autour de certaines personnes connues pour leurs études ou pour leurs fonctions anciennes ou nouvelles ${ }^{5}$. Les échanges et débats sont enrichis non par le nombre des usagers, mais plutôt par leur origine sociale diversifiée.

Contrairement à l'origine de l'espace public qui est était bourgeoise chez Habermas, les lieux de vente de vin de palme sont, à notre avis, des espaces publics d'origine prolétaire, eu égard à la qualité des leurs usagers. Ce qui fait la spécificité de ces sites, c'est surtout la présence de différentes couches socioéconomiques dont le revenu reste généralement faible. 


\section{L'administration et le problème de la salubrité dans ces sites}

Parler de l'administration et de la salubrité de ces sites nous pousse à toucher la responsabilité des pouvoirs publics pour ce qui est du droit de regard sur toutes les activités sociales.

En principe, les exploitants de ces sites sont priés, avant de commencer leurs activités, de se présenter au service d'hygiène communal. Ce dernier est chargé du contrôle non seulement de la qualité des denrées alimentaires, mais la bonne santé des vendeurs de ces denrées. Il prodigue des conseils pour maintenir ces espaces propres et, par conséquent, la santé de la population, y compris celle des vendeurs eux-mêmes. Ce service procède normalement à la consultation de l'état physique du vendeur pour voir si sa situation sanitaire n'est pas susceptible de contaminer les autres membres de la société. Ainsi par exemple, si, après examen, l'on constate que le vendeur potentiel ou actif est atteint d'une maladie contagieuse, dont la tuberculose, il lui sera interdit de commencer son activité ou de la poursuivre. Il ne pourra le faire qu'après la guérison.

Malheureusement, ces principes ne sont pas appliqués à cause de l'absence quasi générale de l'Etat et de ses services en République Démocratique du Congo. Chacun peut se constituer en exploitant ou en vendeur de vin de palme et mettre fin à cette activité quand il veut, sans informer qui que ce soit.

Les conditions hygiéniques dans lesquelles cette boisson est consommée ne font pas douter qu'elles ne sont pas acceptables. Ces endroits attirent bien des moustiques et les récipients dans lesquels ce vin est consommé ne sont pas du tout très propres. Ce qui traduit la misère de la population et la crise de tout contrôle de salubrité. Des mouches entrent même dans les récipients et les consommateurs n'en font pas du tout un problème. Cela peut constituer une cause de maladies telles que la fièvre typhoïde et la diarrhée.

Ces espaces peuvent constituer des foyers de certaines maladies dont les conséquences peuvent s'avérer graves pour la vie de toute la population. L'Etat ne se préoccupe ni de l'hygiène, ni de la salubrité dans ces sites. Aucune réglementation n'est appliquée à l'endroit des exploitants des ces sites. Cependant, les vendeurs ou exploitants de ces sites se sont organisés pour payer une petite taxe appelée taxe d'étalage à la commune. Cette taxe n'entre même pas dans le compte du trésor public dans la mesure où elle ne demeure pas formalisée.

Ces endroits demeurent des espaces qui doivent normalement attirer l'attention des pouvoirs publics dans la mesure où les conditions de salubrité laissent à désirer. L'impression que donnent ces sites, c'est celle d'un abandon 
des services publics dont la mission première reste la réalisation de l'intérêt général à travers certaines prestations, dont le maintien de l'hygiène et de la salubrité publique.

\section{Des thèmes débattus dans les lieux de consommation de vin de palme}

Il est un constat selon lequel les thèmes qui alimentent les discussions dans ces sites constituent également des facteurs d'attrait des usagers. Nous voulons à présent faire ressortir quelques thèmes qui font la chaleur des échanges dans ces endroits, cela étant les résultats de notre descente sur ces endroits, en tant que chercheur.

En effet, tout est débattu et discuté dans ces endroits. Il n'existe donc pas de sujets qui fassent l'objet de restriction dans ces sites. Toutefois, notre observation démontre bien que les sujets touchant directement les questions d'actualité dans n'importe quel secteur de la vie sont ceux qui sont souvent à l'ordre du jour. Tout dépend des préoccupations de l'heure. A différentes périodes, c'est l'actualité qui conditionne les débats. Tel est le cas de certaines journées qui précèdent ou suivent un match de football qui devrait ou a opposé l'équipe nationale congolaise à une autre sélection nationale. L'on pourrait croire que ces endroits sont devenus des espaces de prise de stratégies pour l'équipe nationale par la nature des échanges qui s’y passent. Loin de cela, il est plus question de la place qu'occupe l'actualité dans les débats organisés dans ces endroits.

Au-delà des questions d'actualité, signalons également que les problèmes de société font également partie des débats et échanges. Ainsi par exemple, l'habillement des jeunes filles, le comportement de certains prêtres et pasteurs, la corruption, sans oublier la musique. Cette dernière reste, avec le football, un des piliers de l'identité congolaise (Kayser 2004:10).

Si tout thème peut faire l'objet de débats et discussions, il convient de faire remarquer que tous les sujets n'attirent pas de la même façon ou encore ne captivent pas les usagers de la même manière. Aussi tous les usagers ne sont-ils pas attirés de la même manière par tel ou tel autre thème développé. Toutefois, il sied de préciser que les thèmes à caractère politique intéressent plus les usagers que les autres thèmes. Pendant les élections par exemple, c'est dans ces endroits qu'on pouvait bien entendre les débats et discussions sur le projet de société de tel ou tel parti, en dépit du fait que ceux qui prenaient la parole le faisaient souvent par militantisme aveugle. Avec la situation politico-militaire qui prévaut à l'Est du pays dans les provinces du nord et sud Kivu, il ne se passe pas une journée dans ces lieux sans qu'on soulève une question touchant à cette situation d'insécurité. 
Les amateurs de vin de palme, toutes catégories confondues, sont conscients du fait que leur vie dépend de la façon dont la politique est menée par le gouvernement. Pour eux, parler politique a plus d'intérêt que de parler des autres secteurs de la vie. Quant aux fonctionnaires, plus particulièrement, ces endroits constituent des espaces leur permettant de s'exprimer en toute liberté, car ils peuvent y défendre certaines opinions sans faire prévaloir le devoir de réserve auquel ils sont soumis. En effet, ils sont appelés à manifester une certaine neutralité pendant qu'ils sont au service. Ces sites constituent pour eux des endroits idéaux pour s'exprimer sans aucune contrainte. Ils ne sont donc tenus au respect d'aucun principe.

Les sans-emploi et les étudiants trouvent également ces endroits propices à la libre expression et aux échanges sincères. Les sans-emploi estiment que seuls ces sites leur permettent de parler et de se faire entendre; l'accès aux médias n'est pas donné à tout le monde et, surtout tout ne peut pas être dit dans les médias.

Du point de vue genre, nos investigations ont démontré que les hommes sont plus nombreux que les femmes dans ces sites. Cela ne veut pas nécessairement dire que les femmes consomment moins le vin de palme que les hommes. Cette faible représentation du sexe féminin peut se justifier pour les raisons diverses touchant aux heures d'ouverture de ces sites qui sont, avons-nous dit, les heures du soir. A ces moments-là, les femmes s'adonnent plus à la cuisine après une journée chargée de quête de la nourriture. Aussi les femmes qui pouvaient se présenter dans ces lieux publics seraient-elles moins considérées que celles qui pourraient prendre leur bière dans les bars ordinaires.

Quelles sont alors les sources des informations qui alimentent les débats dans ces sites?

\section{Sources et problème de la dénaturalisation des informations}

En dépit du pluralisme médiatique qui caractérise l’univers politique congolais, il est utile de signifier que les médias ne fonctionnent pas correctement du fait de leur coloration politique ou sociale. Ainsi, la radiotrottoir trouve un espace favorable pour son développement. De ce fait, les radios et les télévisions ainsi que les journaux sont les principales sources d'informations qui alimentent les débats dans ces lieux.

L'Internet constitue actuellement une des principales sources qui font également autorité dans ces sites. A propos de l'Internet, il convient de faire remarquer que son importance se comprend par le degré de confiance que certaines personnes ont vis-à-vis de ces sources. Cela s'explique par le fait que la majorité de la population n'a pas accès à l'Internet et que l'on croit ce 
moyen moderne de communication fiable par rapport aux autres. Aussi le fait que les mécanismes de fonctionnement de l'Internet ne soient pas connus par certaines personnes fait-il passer cette source pour une source sûre et par conséquent digne de foi. Ainsi, lors des débats par exemple, il suffit de citer l'Internet comme source pour que les autres interlocuteurs vous prennent au sérieux, ce qui n’est pas nécessairement le cas en réalité. Car, actuellement, certaines informations véhiculées par l'Internet restent de simples spéculations.

Les informations livrées par les chaines de radio et télévision sont souvent dénaturées dans ces sites du fait de leur transmission orale. L'oralité reste la voie privilégiée de communication dans ces endroits, avec toutes ses faiblesses. L'expression " on a dit » revient souvent dans les échanges communicationnels, l'oralité étant comprise comme étant la propriété d'une communication réalisée sur la base privilégiée d'une perception auditive du message (Calvet 1984:6).

Les milieux de vente de vin de palme sont de véritables foyers de dénaturalisation des informations. Les informations livrées sont souvent entachées de beaucoup de subjectivité, ce qui rend de plus en plus les débats chaleureux à cause des interventions souvent spontanées de chacun des usagers. A côté des informations officielles, les commentaires personnels dénaturent davantage le contenu des informations tirées des sources conventionnelles que sont les médias.

Faisons remarquer que l'organisation des discussions dans ces lieux se caractérise par une spontanéité telle que l'on pourrait se croire dans une organisation structurée. L'absence d'un modérateur n'enlève en rien la disponibilité d'écoute et de patience de la part des différents interlocuteurs. Quelquefois, il existe l'improvisation d'un modérateur qui souvent est accepté sans aucune contestation, chacun voulant pour cela voir les discussions continuer dans un climat d'apaisement et de tolérance. Cela ne veut donc pas dire qu'il n’y a pas de dérapage dans ces endroits.

\section{De la banalité à l'expression de l'opinion publique}

De façon simple, nous pouvons affirmer que les sites de vin de palme demeurent à première vue des endroits moins intéressants en ce qui concerne l'expression politique. Cependant, l'approche de la politologie de la quotidienneté relève que ces points de vente font fonction d'endroits où l'on peut, avec quelque assurance, savoir ce pensent les gens sur tel ou tel sujet ou telle ou telle autre personnalité politique. On est passé d'un endroit banal à un véritable lieu d'expression d'opinion publique. 
Pendant les périodes de turbulences politiques, le pouvoir essaie, par l'intermédiaire de personnes secrètes, de connaître ce que pense la population en envoyant certains émissaires dans ces endroits de consommation de vin de palme, l'objectif étant ainsi moins d'inquiéter ou d'intimider, mais plutôt de jauger la vraie température de la population. On est en face d'une sorte d'Agora qui ne dit pas son nom. A la différence de l'Agora dans la Grèce antique, les lieux de vente de vin de palme regroupent les citoyens dont la situation économique reste précaire. Le pouvoir reconnaît le caractère libre des débats et échanges qui y ont lieu. Il les tolère sans pourtant le dire expressément. Il y trouve un endroit propice pour s'enquérir réellement de ce qu'est l'opinion publique.

Du côté de l'opposition au pouvoir, ces endroits constituent des canaux par lesquels certains messages peuvent passer au nom de l'amitié de l'ivresse. ${ }^{6}$

La tolérance dont bénéficient ces sites résulte du fait que leurs usagers sont de la catégorie pauvre de la société se considèrent très souvent comme les marginalisés. Le pouvoir en place les interprète dans un sens de défoulement et se refuse de les étouffer, ce qui pourrait constituer un obstacle majeur au minimum de la liberté dont cette tranche de la population peut jouir malgré les conditions économiques très modestes.

De ce qui précède, nous pouvons considérer ces endroits réellement comme des espaces publics sui generis en raison de leur importance dans l'expression de l'opinion à travers les débats et échanges qui y ont lieu autour d'un verre de vin de palme. De la prise du vin de palme (quelque chose de banal), l'on est passé aux discussions et échanges de manière libre, ce que nous appelons l'autre face de l'espace public.

\section{Que conclure?}

Les points vente de vin de palme de vin de palme sont des espaces publics où s'organisent les débats et discussions sur les questions de société tant au niveau local, national qu'international. Ils sont réellement une autre face de l'espace public par le fait qu'ils permettent le libre débat autour de n'importe quel sujet de la société. Ce sont également des espaces où l'on peut connaître ce que pense la population dans sa plus sincère liberté. Ils ne sont plus de simples sites auxquels la notion de banalité doit faire appel à une analyse de la quotidienneté, compte tenu de leur rôle dans la société. Nous pensons que dans la ville comme Kisangani, où il $\mathrm{y}$ a un nombre réduit de débats et conférences, ces sites sont à compter parmi les endroits où se réalise la culture de débats. Le français, qui est la langue officielle, est mis de côté au profit des langues nationales, dont précisément le lingala et le swahili pour la ville de Kisangani. Cela permet à ceux qui ne peuvent pas participer aux 
quelques débats et conférences, qui se tiennent souvent en français, d'avoir droit au chapitre dans un contexte qui est le leur.

Ces endroits s'inscrivent dans les terrains de recherche de la nouvelle génération en science politique. Cette nouvelle génération préconise que les recherches soient tournées vers la rue, la mosquée, le périmètre irrigué, le débit de boissons, le marché, etc. Il n'est point question de ne plus les considérer dans les analyses scientifiques en raison de leur importance dans la communication sociale et surtout politique.

Les conditions difficiles favorisent la multiplicité de ces sites par le fait que le coût de la bière n'est pas à la portée de la bourse des toutes les catégories sociales. En tolérant ces endroits pour de diverses raisons dont nous avons parlé, il convient que les services publics les prennent en charge pour garantir la salubrité. Cette dernière est un des domaines des politiques publiques. Les taxes perçues doivent donc être réglementées pour alimenter le compte du trésor public afin de permettre à l'administration de bien accomplir ses missions, surtout dans le cadre de l'hygiène publique.

\section{Notes}

1. Le vin de palme est appelé libondo en jargon local de Kisangani. Il est extrait après l'abattage de palmier, pour la plupart des cas. Toutefois, l'extraction peut se faire sans abattre le palmier : l'on grimpe sur le bout du palmier et on y extrait la sève.

2. Nous faisons ici allusion à l'analyse fonctionnelle de R. King Merton.

3. Dans les activités journalières, il convient de faire également mention de débrouillardise, qui constitue l'une des principales activités d'un bon nombre de Congolais.

4. Boyoma est un autre nom par lequel les Congolais désignent la ville de Kisangani. Ces habitants sont appelés les Boyomais. Notons également que Kisangani est la troisième ville de la République Démocratique du Congo, compte tenu de son poids économique mais aussi politique.

5. Dans ces lieux, il se crée des relations et des découvertes en ce sens que certaines personnes ont des préférences sur tel ou tel autre site. A force de se voir, on se découvre et l'on se connaît davantage. De même, le fait que certains individus fréquentent certains lieux précis attire d'autres individus qui estiment venir pour se ressourcer en informations ou se constituer en informateurs incontestables.

6. L'amitié de l'ivresse renvoie à une sorte de solidarité qui se manifeste autour d'un verre ou encore entre les fumeurs. Souvent, cette solidarité permet une libre expression et un sentiment de fraternité qui exclut toute traîtrise. 


\section{Bibliographie}

Alcaud, D., et Bouvet, L., éds., 2004, Dictionnaire des sciences politiques et sociales, Paris, Dalloz.

Caillé, A., 2004, Paix et démocratie, une prise de repère, Paris, Centre National des Sciences de l'Homme-Byblos.

Calvet, J.L., 1984, Tradition orale, Paris, PUF.

Kanyarwunga, J., 2008, République Démocratique du Congo. Les générations condamnées. Déliquescence d'une société précapitaliste, Paris, Publibook.

Kayser, Ch., 2004, « Culture et l'interculturation : ressources et richesses dans la lutte contre la fragmentation », Regards Croisés, Goma, Poli institution.

Monga, C., 1994, Anthropologie de la colère, Paris, L'Harmattan.

Steward, F., 1974, Technologie intermédiaire, éléments de définition dans le choix et l'adaptation de la technologie dans les pays en voie de développement, Paris, OCDE. 
